\title{
Laparoscopic Salpingectomy or Hysteroscopic Tubal Occlusion in Patients with Hydrosalpinx and Intracytoplasmic Sperm Injection Outcomes
}

\author{
Ibrahim Mohiy El-Maghraby*, Adel Gaber Allam
}

Department of Obstetrics and Gynecology, Shibin El Kom Teaching Hospital, Egypt

*Corresponding author: Ibrahim Mohiy El-Maghraby, Mobile: (+2)0100035 7896, E-Mail: ibrahim_mohie@yahoo.com

\begin{abstract}
Background: Tubal factor of infertility resulting from various forms of tub peritoneal damage remains an extremely common cause of female infertility. Probably the most severe form of tubal pathology is hydrosalpinx.

Objectives: To compare the efficacy of hysteroscopic tubal electrocoagulation versus laparoscopic salpingectomy for management of hydrosalpinx related infertility among patients undergoing intracytoplasmic sperm injection (ICSI).

Patients and Methods: This retrospective study was conducted at outpatient clinic in Private Infertility Unit, Menoufia, Egypt, from November 2018 to August 2019. This study included 120 infertile patients who were candidate for ICSI and fulfilled inclusion and exclusion criteria, half of the patients underwent hysteroscopic tubal electrocoagulation who have laparoscopic contraindications, while the other half underwent laparoscopic salpingectomy. After tubal occlusion was done, an ICSI cycle was started with assessment of chemical pregnancy rate. Results: The rate of positive chemical pregnancy was $56.16 \%$ in hysteroscopic group and $43.84 \%$ in laparoscopic group. Conclusion: Hysteroscopic tubal electrocoagulation was found to be a successful treatment for hydrosalpinges before ICSI when laparoscopy is contraindicated with chemical pregnancy rate comparable to laparoscopic tubal disconnection.
\end{abstract}

Keywords: Hydrosalpinx, Hysteroscopy, Infertility, Intracytoplasmic sperm injection.

\section{INTRODUCTION}

When the tube is clogged at its distal end or both ends, secretions build up and cause hydrosalpinx. Transient distention of the fallopian tubes might develop due to the retrograde movement of blood from the uterus without total distal blockage on rare instances. Hydrosalpinx is most usually a complication of pelvic inflammatory illness, but it can also occur after tubal ligation for ovulation induction. Other reasons include primary or secondary fallopian tube cancers ${ }^{(1)}$. As a result of a previous pelvic infection, hydrosalpinx is virtually usually the result. Gonorrhea, chlamydia, staphylococcus, streptococcus, and pelvic tuberculosis are the most prevalent causing organisms. ${ }^{(2)}$. The most common treatment options suggested by the clinicians were laparoscopic salpingectomy and tubal disconnection, followed by open salpingectomy, salpingostomy, proximal tubal occlusion, and transvaginal ultrasonographic guided hydrosalpinx aspiration either before or during oocyte retrieval. The proximal closure of hydrosalpinx by hysteroscopic implantation of micro inserts was the most recent treatment option introduced ${ }^{(3)}$.

Tubal ligation-related infertility accounts for $25-35$ percent of female infertility. Hydrosalpinx is a serious type of tubal disease that has been linked to poor pregnancy outcomes in tubal infertility patients (4). Hydrosalpinx diagnosed by trans-vaginal ultrasound had a $10 \%$ prevalence rate, while hydrosalpinx diagnosed via hysterosalpingography (HSG) or laparoscopy had a 30\% prevalence rate. Initially, hysterosalpingography (HSG) was used to diagnose hydrosalpinx, which was then verified by laparoscopy. Later, image diagnosis, such as HSG and ultrasound, was used nearly solely to make the diagnosis ${ }^{(5)}$.
The American Society for Reproductive Medicine (ASRM) suggested laparoscopic fibroblast or neo salpingostomy to treat minor hydrosalpinx in young women without any evident reproductive problems ${ }^{(6)}$. It may be preferable to proceed directly to salpingectomy and IVF treatment in older women or those with more severe hydrosalpinxes. Despite the fact that salpingectomy is the most commonly recommended treatment for hydrosalpinx in clinical practice, other common treatments for hydrosalpinx before IVF include ultrasonic-guided hydrosalpinx aspiration, tubal sclerotherapy, hysteroscopic tubal occlusion, and antibiotic treatment, among others ${ }^{(7)}$. Ultrasonic-guided hydrosalpinx aspiration was found to be less invasive, safer, and easier to conduct under dense adhesion, as well as requiring less time in the hospital. Hydrosalpinx aspiration has also been shown to improve the pregnancy outcome of hydrosalpinx patients, and it is used in clinical practice. Before IVF, hysteroscopic occlusion was also found to be an effective treatment for hydrosalpinx in women ${ }^{(8)}$. Although laparoscopic salpingectomy was the preferred way of surgical therapy, over half of Society for Reproduction Endocrinology and Infertility (SREI)/Society of Reproductive Surgeons (SRS) members said hysteroscopic tubal occlusion should have a role as a major mode of intervention ${ }^{(9)}$. With the benefits of hysteroscopic operations such as speedier recovery, fewer hospitalisation, and quicker return to work, it may be done in the outpatient clinic as an office surgery in the future. In order to examine the efficacy of hysteroscopic tubal electrocoagulation vs laparoscopic salpingectomy for the treatment of hydrosalpinx-related infertility in patients having surgery, researchers conducted this study. 


\section{PATIENTS AND METHODS}

A retrospective study was conducted at an outpatient clinic in the Private Infertility Unit in Menoufia, Egypt, from November 2018 to August 2019. The study included 120 infertile patients who were receiving ICSI and had unilateral or bilateral hydrosalpinges as determined by hysterosalpingography and/or vaginal ultrasonography. The patients were separated into two groups: 60 had laparoscopic salpingectomy (Group (I)) and 60 had hysteroscopic tubal electrocoagulation (Group (II)).

Inclusion criteria: Women between the ages of 18 and 41 who were candidates for ICSI because of firsttrimester infertility or second-trimester infertility with hydrosalpinx, as determined by hysterosalpingography (HSG) and/or transvaginal ultrasound. Patients with uterine factor infertility, such as fibroids (Type 0 or 1 ), patients with male factor infertility, and patients with tubal blockage were all asked to complete the following questionnaire: Infertility duration and type (primary or secondary), sexual history, history suggestive of pelvic inflammatory illness, past history of pelvic surgery, and history of chronic diseases are all taken into consideration.

Infertility clinic specialists did a clinical general and local examination, including weight, height, BMI, thyroid examination, local bimanual examination (tenderness or lumps), and speculum examination.

All of the patients had been diagnosed with infertility (FSH, LH, estradiol serum level, prolactin and $\mathrm{TSH}$, husband seminal analyses, routine labs in the form of: Complete blood count, Rh typing, urine analysis, random blood glucose level, liver and kidney functions and coagulation profile). The uterus was scanned in the sagittal plane for any endometrial abnormalities, and visible hydrosalpinx in the form of an extended, diluted, tortuous tube holding fluid that was anechoic was recorded using pelvic sonography. Hydrosalpinx appears on ultrasound as a tubular shape with an echogenic wall, folded configurations, and linear echos in the fallopian tube lumen.

Under general anesthesia, the pneumoperitoneum was produced using a Veress needle using carbon dioxide and the pressure was held at 12 $\mathrm{mmHg}$ for half of the patients to confirm the presence of the hydrosalpinx, and unilateral or bilateral salpingectomy was performed when technically viable. For endoscopic visualization of the abdominal cavity, a $2 \mathrm{~cm}$ trans-umbilical incision was made and a $10 \mathrm{~mm}$ reusable umbilical optical trocar was introduced. In all cases, a uterine sound was introduced as a uterine manipulator to allow proper exposure of the pelvic organs. In the midline, a $10 \mathrm{~mm}$ trocar was placed $3 \mathrm{~cm}$ above the symphysis pubis. Bipolar diathermy was applied as close to the tube as possible. Because the blood supply to the ovaries and tubes are in close proximity with various degrees of anastomosis, special care was taken not to disturb the ovarian blood supply during salpingectomy followed by cutting of the diathermies point with scissors. The other half of the patients who were not candidates for laparoscopy had hysteroscopic tubal electrocoagulation in the second week of their cycle, using Essure intratubal devices for hysteroscopic fulguration of internal orifices of fallopian tubes at a hysteroscopic unipolar coagulation power of $50 \mathrm{w}$ for 20 seconds, resulting in degeneration of diseased tube internal orifice tissue by electric heat energy to form tissue scar so as to prevent hydrosalpinx fluid reflux to uterine cavity, helping embryo's development and implantation .

Following tubal occlusion, an ICSI cycle was initiated as follows: Protocol for ovulation stimulation from day 21 of the stimulation cycle, the patient was treated with pituitary desensitization using a gonadotrophin releasing hormone $(\mathrm{GnRH})$ agonist. Patients were then given 150-300 units of human menopausal gonadotrophin (HMG) injections daily from the second to third day of their menstrual cycle. A 10,000 IU unit of HCG was given when at least three follicles had a diameter more than 16-18 $\mathrm{mm}$ and the estradiol content was acceptable. Transvaginal ultrasonography guided ovum pick up was performed 34-36 hours after HCG injection. Following that, only metaphase II oocytes with the first polar body were chosen for fertilization. 3-6 hours after oocyte recovery, and 48-72 hours later, ICSI was conducted. Serum B-hCG test was done to confirm pregnancy two weeks after embryo transfer (chemical pregnancy).

\section{Ethical consent:}

An approval of the study was obtained from Shibin El Kom Teaching Hospital Academic and Ethical Committee. Every patient signed an informed written consent for acceptance of the participation in the study after explaining the nature and scope of the study to all of them. This work has been carried out in accordance with The Code of Ethics of the World Medical Association (Declaration of Helsinki) for studies involving humans.

\section{Statistical analysis}

Results were collected, tabulated and statistically analyzed by an IBM compatible personal computer with SPSS statistical package version 25 (SPSS Inc. IBM SPSS statistics for windows, version 25, Armonk, NY: IBM Corp, USA). Categorical baseline data were reported as absolute numbers and percentages and were compared by Chi square test. Normally distributed continuous variables were summarized as means with standard deviations (SDs) and non-normally distributed continuous variables were reported as medians with $25^{\text {th }}-75^{\text {th }}$ percentiles. For normally distributed continuous outcomes, mean differences were calculated and independent t-test was used. For non-normally distributed continuous outcomes Mann-Whitney U-test was used. A value of P 0.05 was considered significant. 


\section{RESULTS}

There was statistically significant difference between the two groups in terms of primary diagnosis (Table 1).

Table (1): Demographic details and diagnosis of the two studied groups

\begin{tabular}{|c|c|c|c|}
\hline Characteristics & $\begin{array}{c}\text { Group (I) } \\
(\mathrm{N}=60)\end{array}$ & $\begin{array}{c}\text { Group (II) } \\
(\mathrm{N}=60)\end{array}$ & Sig. \\
\hline Age (Years) & $32.44 \pm 4.73$ & $32.94 \pm 4.73$ & 0.564 \\
\hline BMI $\left(\mathrm{kg} / \mathrm{m}^{2}\right)$ & $29.88 \pm 4.63$ & $29.89 \pm 4.78$ & 0.960 \\
\hline $\begin{array}{l}\text { Infertility type: } \\
\text { Primary }\end{array}$ & $42(70.00 \%)$ & $41(70.00 \%)$ & \multirow[t]{2}{*}{0.843} \\
\hline Secondary & $18(30.00 \%)$ & $19(30.00 \%)$ & \\
\hline Duration of infertility (year) & $5.30 \pm 2.62$ & $5.0 \pm 2.58$ & 0.592 \\
\hline $\begin{array}{l}\text { Primary diagnosis: } \\
\text { Unilateral } \mathrm{Hx} \\
\text { Bilateral } \mathrm{Hx}\end{array}$ & $\begin{array}{l}25(41.67 \%) \\
35(58.33 \%)\end{array}$ & $\begin{array}{l}38(63.33 \%) \\
22(36.67 \%)\end{array}$ & 0.017 \\
\hline
\end{tabular}

Values are given as mean \pm SD or number (percentage). HX: hydrosalpinX

$\mathbf{N}$ : number, $\mathbf{k g} / \mathbf{m}^{2}$ (kilograms per square meter)

There was no statistically significant difference between the two groups in terms of pretreatment hormonal profile (Table 2).

Table (2): Hormonal profile of the two studied group

\begin{tabular}{|l|c|c|c|}
\hline Hormones & $\begin{array}{c}\text { Group1 } \\
\text { (N=60) }\end{array}$ & $\begin{array}{c}\text { Group2 } \\
\text { (N=60) }\end{array}$ & Sig. \\
\hline FSH $(\mathrm{IU} / \mathrm{ml})$ & $6.81 \pm 1.55$ & $7.14 \pm 1.60$ & 0.521 \\
\hline $\mathrm{LH}(\mathrm{IU} / \mathrm{ml})$ & $4.49 \pm 1.47$ & $4.41 \pm 1.96$ & 0.865 \\
\hline Prolactin(ng/ml) & $16.88 \pm 3.64$ & $17.88 \pm 3.56$ & 0.566 \\
\hline Estradiol $(\mathrm{pg} / \mathrm{ml})$ & $55.82 \pm 5.47$ & $57.59 \pm 3.79$ & 0.721 \\
\hline AMH $(\mathrm{ng} / \mathrm{ml})$ & $2.85 \pm 0.88$ & $2.66 \pm 0.75$ & 0.603 \\
\hline TSH $(\mathrm{miu} / \mathrm{ml})$ & $2.58 \pm 0.13$ & $2.47 \pm 0.14$ & 0.634 \\
\hline
\end{tabular}

FSH (Follicular stimulating hormone), LH (luteinizing hormone), AMH (Anti Mullerian hormone), TSH (Thyroid stimulating hormone), IU (International unit), ng (nanogram), Pg (picogram), ml (milliliter), miu (milli International unit), Data are presented as mean \pm standard deviation

During oocyte retrieval, there was no statistically significant difference in the total number of oocytes between the two groups. These ova were mostly in mature metaphase I and II, with no statistically significant difference in the number of MI oocytes or MII oocytes between the two groups. In terms of overall number of embryos, there was no statistically significant difference between the two groups (Table 3,4).

Table (3): Number of ova retrieved and number of embryos transferred among study participants

\begin{tabular}{|l|c|c|c|}
\hline Variable Group & $\begin{array}{c}\text { Group1 } \\
\text { N=60 }\end{array}$ & $\begin{array}{c}\text { Group2 } \\
\mathbf{N = 6 0}\end{array}$ & Sig. \\
\hline Total No Ova Retrieved & $12.2 \pm 2.6$ & $12.01 \pm 2.6$ & 0.389 \\
\hline N. of M II oocytes & $8.98 \pm 2.06$ & $8.99 \pm 2.6$ & 0.094 \\
\hline N. of M I oocytes & $1.04 \pm 1.1$ & $1.02 \pm 0.6$ & 0.084 \\
\hline N. of GV oocytes & $1.07 \pm 0.75$ & $0.81 \pm 0.894$ & 0.332 \\
\hline N. of Atretic ova & $0.01 \pm 0.38$ & $0.2 \pm 0.5$ & 0.812 \\
\hline
\end{tabular}

GV: germinal vesicle phase, MI: metaphase I, MII: metaphase II, N: number, Data presented as median \pm SD 
Table (4): Total embryos and grades of produced embryos in the two studied group

\begin{tabular}{|l|c|c|c|}
\hline \multicolumn{1}{|c|}{ Group } & $\begin{array}{c}\text { Group I } \\
\text { N=60 }\end{array}$ & $\begin{array}{c}\text { Group II } \\
\text { N=60 }\end{array}$ & P-Value \\
\hline Total number of embryos produced & $\begin{array}{c}3.50 \pm 1.05 \\
7(5-10)\end{array}$ & $\begin{array}{c}3.50 \pm 2.76 \\
7(4-10)\end{array}$ & 0.673 \\
Mean \pm SD & $\begin{array}{c}2.00 \pm 2.50 \\
(1-3)\end{array}$ & $\begin{array}{c}2.00 \pm 2.50 \\
(1-3)\end{array}$ & 1.000 \\
\hline $\begin{array}{l}\text { Totian (range) } \\
\text { Mean } \pm \text { SD }\end{array}$ & & \\
Range & & \\
\hline
\end{tabular}

The proportions of pregnancies in the two groups were comparable; 37 pregnancies were identified chemically in the laparoscopic disconnection group versus 29 in the hysteroscopic group (Table 5).

Table (5): Proportion of pregnancies in the two studied groups

\begin{tabular}{|l|c|c|}
\hline \multicolumn{1}{|c|}{ Variable } & $\begin{array}{l}\text { Positive } \\
\text { Group }\end{array}$ & $\begin{array}{c}\text { Negative } \\
\text { (T2=54) }\end{array}$ \\
\hline Group (I) $(\mathrm{N}=60)$ & $37(56.06 \%)$ & $23(42.59 \%)$ \\
\hline Group2 (N=60) & $29(43.94 \%)$ & $31(57.41 \%)$ \\
\hline P- value & \multicolumn{2}{|c|}{0.142} \\
\hline
\end{tabular}

Values are given as number (percentage)

\section{DISCUSSION}

When laparoscopy is contraindicated, hysteroscopic tubal electrocoagulation was found to be an effective treatment for hydrosalpinxes before ICSI, with a pregnancy rate comparable to laparoscopic salpingectomy. The success rate of hysteroscopic tubal electrocoagulation for the treatment of hydrosalpinxrelated infertility in patients with laparoscopic contraindications undergoing ICSI was investigated in this retrospective clinical study. The patients were divided into two groups: 60 patients underwent laparoscopic salpingectomy (Group (I)) and 60 patients underwent hysteroscopic tubal occlusion (Group (II). In Group (I), 37 (56.16 percent) of 60 cases were pregnant, whereas in Group (II), 29 (43.84 percent) of 60 cases were pregnant, with no significant difference between the two groups.

Before ICSI, hysteroscopic tubal electrocoagulation could be a viable option for hydrosalpinx therapy. Hysteroscopic surgery, on the other hand, is linked to the risk of electrosurgical damage as well as distending media problems. Electrosurgical injury can be avoided with proper insulation and the lowest possible power setting, whereas distending media complications can be avoided with proper fluid input and output monitoring, as well as keeping the uterine cavity distention pressure below the mean arterial pressure to avoid fluid and electrolyte disturbances. There is enough data to suggest that hydrosalpinx fluid in the uterine cavity has a deleterious influence on female fecundity, as well as that blockage of the hydrosalpinx improves fertility significantly ${ }^{(\mathbf{1 0})}$. Although laparoscopic techniques (salpingectomy or proximal tubal disconnection) have been shown to be effective, the cumulative effect of these surgeries is still unknown, the cumulative risks for patients undergo ICSI treatment are a concern. Hysteroscopic procedures (either an Essure insert or electrocoagulation) are regarded as simpler and safer ${ }^{(\mathbf{1 1})}$.

The first trial of hysteroscopic tubal electrocoagulation as a technique for tubal occlusion in cases of hydrosalpinx was evaluated in a pilot study. Rosen et al. ${ }^{(12)}$ only employed the Essure set for tubal occlusion in instances with hydrosalpinx in a case report, and it was a successful trial, although this method is more expensive than the one, we used. Aboulghar et al. ${ }^{(13)}$ evaluated hysteroscopic roller ball and needle electrode coagulation of the cornual end of the tubes for blockage of a communicating hydrosalpinx in ten IVF patients in a pilot study. One tube was successfully closed in the roller ball group (6 tubes/4 patients). One tube was successfully closed, three tubes were found to be partially open, and two tubes were discovered to be entirely open. The needle electrode group (10 tubes/ 6 patients) achieved a $90 \%$ occlusion success rate (only one tube was found to be open). However, that study only included a small number of cases. El-Mazny et al. ${ }^{(14)}$ conducted a prospective clinical trial to determine the success rate of hysteroscopic tubal electrocoagulation for the treatment of hydrosalpinx-related infertility in a large number of patients having IVF with surgical contraindications. Overall, 25 (93 percent) of 27 hydrosalpinxes in the hysteroscopic group and 78 (96 percent) of 81 hydrosalpinxes in the laparoscopic group had tubal blockage, with no significant difference between the two groups. These results are close to our study results 
and that may be due to some common points that were used in both studies, as all the hysteroscopic procedures in both studies were performed in the early follicular phase and the instruments used were close to each other, as they used in this previous study the electrocoagulation roller ball as we used in our study.

\section{CONCLUSION}

Hydrosalpinx can be treated surgically before ICSI cycles by occluding the cornual end of the afflicted tube or performing a laparoscopic salpingectomy. Because the two procedures are generally safe and have similar effectiveness and chemical pregnancy rates, proximal closure of a hydrosalpinx by hysteroscopy may be a viable treatment option when laparoscopy is technically challenging or contraindicated.

\section{Financial support and sponsorship: Nil. \\ Conflict of interest: Nil.}

\section{REFERENCES}

1. Chen C, Yang J, Lin K et al. (2002): The significance of cytokines, chemical composition, and murine embryo development in hydrosalpinx fluid for predicting the IVF outcome in women with hydrosalpinx. Human Reproduction, 17(1):128-33.

2. Fikry M, Hamam M, Faisal M et al. (2016): Endometritis in hydrosalpinx and laparoscopic salpingectomy versus laparoscopic tubal disconnection in cases of hydrosalpinx regarding the effect on ovarian stromal blood flow and outcome of intracytoplasmic sperm injection. World Journal of Medical Sciences, 13(2):139-44

3. Hammadieh N, Afnan M, Evans J et al. (2004): A postal survey of hydrosalpinx management prior to IVF in the United Kingdom. Human Reproduction, 19(4):1009-12.

4. Melo P, Georgiou E, Johnson N et al. (2020): Surgical treatment for tubal disease in women due to undergo in vitro fertilisation. https://www.researchgate.net/publication/345718632_S urgical_treatment_for_tubal_disease_in_women_due_to _undergo_in_vitro_fertilisation

5. Ng K, Cheong Y (2019): Hydrosalpinx-salpingostomy, salpingectomy or tubal occlusion. Best Practice and Research Clinical Obstetrics and Gynaecology, 59: 41-7

6. Schlaff W (2019): A reconsideration of salpingectomy for hydrosalpinx before in vitro fertilization: why bother. Fertility and Sterility, 111(4):650-1.

7. Volodarsky-Perel A, Buckett W, Tulandi T (2019): Treatment of hydrosalpinx in relation to IVF outcome: a systematic review and meta-analysis. Reproductive Biomedicine Online, 39(3):413-32.

8. Zhou Y, Jiang H, Zhang W et al. (2016): Ultrasoundguided aspiration of hydrosalpinx occurring during controlled ovarian hyperstimulation could improve clinical outcome of in vitro fertilization-embryo transfer. Journal of Obstetrics and Gynaecology Research, 42(8):960-5.

9. Ersahin A, Ersahin S, Gungor N (2020): Surgical removal of hydrosalpinx improves endometrium receptivity by decreasing nuclear factor-kappa B expression. Reproductive Sciences, 27(3):787-92.

10. Tsiami A, Chaimani A, Mavridis D et al. (2016): Surgical treatment for hydrosalpinx prior to in-vitro fertilization embryo transfer: a network meta-analysis. Ultrasound Obstet Gynecol., 48(4):434-445.

11. Dreyer $K$, Lier $M$, Emanuel $M$ et al. (2016): Hysteroscopic proximal tubal occlusion versus laparoscopic salpingectomy as a treatment for hydrosalpinges prior to IVF or ICSI: an RCT. Hum Reprod., 31(9):2005-16.

12. Rosen eld R, Stones R, Coates A et al. (2005): Proximal occlusion of hydrosalpinx by hysteroscopic placement of microinsert before in vitro fertilization-embryo transfer. Fertility and Sterility, 83(5):1547-e11.

13. Aboulghar M, Azmy O, Nada A et al. (2008): Hysteroscopic tubal electrocoagulation in cases with communicating hydrosalpinx and planning for IVF-a pilot study. Int J Med., 1(2):81-5.

14. El-Mazny A, Abou-Salem N, Hammam $M$ et al. (2015): Hysteroscopic tubal electrocoagulation versus laparoscopic tubal ligation for patients with hydrosalpinges undergoing in vitro fertilization. International Journal of Gynecology and Obstetrics, 130(3):250-2. 